Published in final edited form as:

Nat Rev Microbiol. 2013 October ; 11(10): 667-673. doi:10.1038/nrmicro3110.

\title{
Phenol-soluble modulins and staphylococcal infection
}

\author{
Andreas Peschel ${ }^{1}$ and Michael Otto ${ }^{2,}$ \\ ${ }^{1}$ Cellular and Molecular Microbiology Division, Interfaculty Institute of Microbiology and Infection \\ Medicine, University of Tubingen, Germany \\ 2Pathogen Molecular Genetics Section, Laboratory of Human Bacterial Pathogenesis, National \\ Institute of Allergy and Infectious Diseases, The National Institutes of Health, Bethesda, MD, USA
}

\begin{abstract}
Staphylococcus aureus is an important human pathogen and a leading cause of death worldwide. Phenol-soluble modulins (PSMs) have recently emerged as a novel toxin family defining the virulence potential of highly aggressive $S$. aureus isolates. PSMs have multiple roles in staphylococcal pathogenesis, causing lysis of red and white blood cells, stimulating inflammatory responses and contributing to biofilm development and the dissemination of biofilm-associated infections. Moreover, the pronounced capacity of PSMs to kill human neutrophils after phagocytosis may explain failures in anti-staphylococcal vaccine development. Here, we will review the biochemical and genetic properties of PSMs and their role in S. aureus pathogenesis, and suggest potential avenues to target PSMs for anti-staphylococcal drug development.
\end{abstract}

Several members of the genus Staphylococcus can cause disease and Staphylococcus aureus in particular is an extremely virulent pathogen. Every human being is colonized with Staphylococcus epidermidis and a series of other staphylococci. In contrast, S. aureus colonizes only about one third of the population, mainly in the nose, and carriage of $S$. aureus predisposes to infection ${ }^{1}$. S. aureus is the causative agent of a series of diseases ranging from moderately severe skin infections to fatal necrotizing pneumonia and one of the most frequent causes of morbidity and mortality throughout the world ${ }^{2}$. It has been estimated that in the United States, S. aureus infections cost more lives than HIV/AIDS ${ }^{3}$. Other species such as S. epidermidis are commonly opportunistic and cause chronic disease $^{4}$, which often proceeds with the involvement of biofilms, sticky, cellular agglomerations that protect from antibiotics and host defenses ${ }^{5}$. Notably, frequent antibiotic resistance and the lack of an FDA-approved vaccine strategy severely complicate treatment of staphylococcal infections.

The virulence potential of $S$. aureus is to a large extent defined by its capacity to produce a plethora of different toxins, many of which target mechanisms of host defense ${ }^{6}$. Some $S$. aureus toxins are encoded on mobile genetic elements (MGEs) and are thus limited to a subset of $S$. aureus isolates, whereas others are encoded on the $S$. aureus core genome or on highly conserved genomic islands and so are produced by virtually all strains. Among the

Corresponding author: Address, 9000 Rockville Pike, Bldg 33, Bethesda, MD 20892, USA; Phone +1 302443 5209; Fax +1 301480 3632; motto@niaid.nih.gov. 
latter, phenol-soluble modulins (PSMs) have recently attracted much attention, because they have been found to have a key impact on the pathogenesis of $S$. aureus infections ${ }^{7,8}$.

PSMs were first identified in 1999 with the description of a "pro-inflammatory complex" isolated by hot phenol extraction from S. epidermidis culture filtrate, in which three peptides termed "phenol-soluble modulin (PSM)" $a, \beta$, and $\gamma$ were identified ${ }^{9}$. PSM $\gamma$ is identical to the previously described $S$. epidermidis $\delta$-toxin ${ }^{10}$, which is the preferred term. While more recent analysis of PSM-receptor interactions ${ }^{11}$ suggests that the TLR2-stimulating capacities attributed to PSMs in the initial studies $9,12-14$ were likely caused at least in part by impurities, this paved the way for further investigation.

S. aureus PSMs were subsequently identified that killed human neutrophils and had a major impact on the ability of the recently emerged community-associated methicillin-resistant $S$. aureus (CA-MRSA) strains to cause disease ${ }^{7}$. Since then, many reports have been published delineating the roles of PSMs in the pathogenesis and physiology of S. aureus. PSMs were also recognized to contribute to phenotypes not associated with infection, potentially representing the "original" role of PSMs, from which other PSMs evolved as virulence factors ${ }^{15}$. Here, we will focus on the roles of PSMs in staphylococcal infection and discuss the mechanisms underlying their contribution to pathogenesis. We will also discuss how PSMs could be targeted for anti-staphylococcal drug development.

\section{PSMs: genetic and biochemical properties}

The complement of PSMs in staphylococci varies, with the composition being specific for a given species ${ }^{7,16,17}$. PSM nomenclature is not consistent. In S. epidermidis, they were given consecutive Greek letters upon discovery, whereas in $S$. aureus, they were named with Greek letters according to the grouping of PSMs into the smaller a-type and the larger $\beta$ type group (see below). The latter nomenclature should be kept to designate PSMs discovered in the future. PSM peptides in different species can have the same name but different amino acid sequences and it is therefore best to always refer to a PSM peptide with its name and producing species.

In S. aureus, PSMs are encoded at three different locations in the genome ${ }^{7}$. Four PSMa peptides, PSMa1-PSMa4, are encoded in the psma operon; PSM $\beta 1$ and PSM $\beta 2$ are encoded in the $p \sin \beta$ operon; the $\delta$-toxin is encoded within the coding sequence of RNAIII, the effector molecule of the accessory gene regulator (Agr) quorum-sensing system ${ }^{18}$. Other than for S. aureus, the only other staphylococcal species for which there is complete information about the encoded PSM peptides is $S$. epidermidis, which produces the PSMa, $\operatorname{PSM} \beta 1$ and PSM $\beta 2$, PSM $\delta$, PSM $\varepsilon$, and $\delta$-toxin peptides (see Fig. 1 for genomic arrangements and peptide sequences $)^{9}, 19,20$. PSM peptides are grouped according to their length: the a-type peptides (S. aureus PSMa1-a4, S. epidermidis PSMa, $\delta, \varepsilon$, and the $S$. aureus and $S$. epidermidis $\delta$-toxins) are $\sim 20-25$ amino acids long, while the $\beta$-type peptides (S. aureus and S. epidermidis PSM $\beta 1$ and PSM $\beta 2$ peptides) are $\sim 44$ amino acids in length.

Other than for PSM peptides encoded together in one operon, which apparently arose from gene duplication events, the amino acid sequence similarity of PSM peptides is limited. Rather, PSM peptides are grouped together by their physico-chemical properties. While 
originally defined by their behavior during hot phenol extraction, today PSMs are identified by elution at exceptionally high concentrations of organic solvent during reversed-phase chromatography. Such elution is possibly due to the amphipathic a-helix that all PSM peptides form, stretching over virtually the entire length of the peptide in the shorter a-type PSMs and located in the C-terminal region in the longer $\beta$-type PSMs (Fig. 1). PSMs do not have uniform charge characteristics. PSM $\beta$ peptides of $S$. aureus and S. epidermidis are all negatively charged, while many a-type PSMs are positively charged, and the $\delta$-toxin in both species is neutral (all in their N-formylated form, see Fig. 1).

The characteristic surfactant-like properties that are caused by the pronounced amphipathy of PSMs are reflected for example by the tendency of PSMs to aggregate in oligomers, the capacity to facilitate spreading on surfaces ${ }^{21}$ or structure biofilms ${ }^{22,23}$. These features likely play a role in promoting colonization of the skin and emulsifying nutrients in that environment, and may constitute the "original" purpose of PSMs, before some PSMs evolved to fulfill roles in pathogenesis e.g. by disrupting primitive phagocytes ${ }^{15}$.

Some PSM-like peptides had been previously reported in other staphylococcal species. For example, the slush peptides of Staphylococcus lugdunensis ${ }^{24}$ and the gonococcal growth inhibitor peptides from Staphylococcus haemolyticus ${ }^{25}$ are now known to belong to the PSM $\beta$ family. Several other PSM peptides in staphylococcal species other than S. aureus and S. epidermidis were found by liquid chromatography/mass spectrometry, but their amino acid sequences and encoding genes have not yet been identified ${ }^{17}$.

Notably, for many of the biological functions of PSMs described in the following, it is important that they are under exceptionally strict and direct regulation by the Agr quorumsensing system, promoting strong enhancement of PSM production at high cell density 26,27 (Fig. 2). In contrast to most other genes in the Agr regulon, which are regulated via RNAIII, binding of the AgrA response regulator protein directly enhances transcription of the psm $\operatorname{loci}^{28}$.

The PSM-mec peptide, which is produced by many MRSA and MRSE (methicillin-resistant S. epidermidis) strains, represents an exception to the rule that PSM peptides are encoded on the core genome. This PSM is encoded adjacent to the mecA/R/I gene cluster in SCCmec (staphylococcal cassette chromosome, a MGE conferring methicillin resistance) types II, III, and VIII ${ }^{29}, 30$. Interestingly, similar to the RNAIII-encoded $\delta$-toxin, the coding sequence for PSM-mec is embedded within a regulatory $\mathrm{RNA}^{31}$, one of whose functions is to inhibit translation of $\mathrm{AgrA}^{32}$, resulting in decreased expression of other PSMs. However, this activity is highly strain-dependent ${ }^{30,32}$.

\section{PSM export}

All PSMs are secreted without a signal peptide, carrying an $\mathrm{N}$-terminal $\mathrm{N}$-formyl methionine. The formyl group can be removed by cytosolic $\mathrm{N}$-deformylase, dependent on culture conditions ${ }^{33}$, which appears to happen to a more pronounced extent in S. aureus than S. epidermidis ${ }^{16}$. Lack of a signal peptide indicates a dedicated mechanism of secretion, which was recently identified to be a four-component ABC transporter ${ }^{34}$ (Fig. 3). This transporter plays an essential role in $S$. aureus physiology, because in its absence PSM 
peptides accumulate in the cytosol, leading to cell death. The transporter, named Pmt (phenol-soluble modulin transporter) secretes all PSMs in S. aureus; the presence of Pmt homologues in other staphylococcal species indicates a conserved role for Pmt in PSM secretion among the staphylococci. Interestingly, there are no Pmt homologues in other genera. This may explain why PSM production is limited to the genus Staphylococcus: due to the essentiality of the transporter in the presence of PSM production, evolution of PSM peptides appears to have only been possible with the presence or co-evolution of an efficient secretion system.

\section{PSMs and pathogenicity}

The PSMa peptides of $S$. aureus have a key impact on the capacity of virulent $S$. aureus to cause skin infection and bacteremia in animal infection models ${ }^{7,8}$. This was demonstrated using the CA-MRSA strains USA300 and USA400, MRSA strains with increased virulence, as compared to hospital-associated MRSA, that have emerged during the last decade and can infect otherwise healthy individuals ${ }^{35}$. The $\delta$-toxin had a moderate effect and the PSM $\beta$ peptides no effect in those studies ${ }^{7}$. Furthermore, all $S$. aureus PSM peptides and the $S$. epidermidis PSM $\beta$ peptides facilitate the dissemination of biofilm-associated infection to other organs in the human body 22,23 . Other $S$. epidermidis psm loci have not yet been assayed for their contribution to virulence using deletion mutants. Together, these findings indicate that PSM peptides have a strong impact on the pathogenesis of major staphylococcal disease types and have undergone divergent evolution to develop specific functions in pathogenesis.

\section{PSM-mediated cytolysis}

The ability to lyse eukaryotic cells is arguably the most important contribution of PSMs to $S$. aureus pathogenesis (Fig. 4). It is likely receptor-independent ${ }^{11}$, thus targeting virtually every eukaryotic cytoplasmic membrane and distinguishing PSMs from other cytolytic $S$. aureus toxins, such as a-toxin or the bicomponent leukocidins, which are often highly specific for a particular cell type and host species ${ }^{36}$. Not all S. aureus PSMs are cytolytic however. The PSMa peptides of $S$. aureus have a pronounced ability to lyse human leukocytes and erythrocytes, with PSMa3 having by far the strongest activity 7,37 , the $\delta$ toxin has moderate cytolytic activity and the PSM $\beta$ peptides are non-cytolytic. These differences seem to correlate at least in part with the charge characteristics of PSMs (Fig. 1), but which structural features of PSMs define their biological activities has not yet been analyzed in detail. Notably, deletion of the psma operon decreases the cytolytic capacity of the highly virulent CA-MRSA strains USA300 and USA400 toward human neutrophils to levels found in strain 252, a standard hospital-associated MRSA strain; and expression of PSMa peptides or PSMa3 alone at physiological levels in strain 252 leads to cytolytic capacity similar to that found in CA-MRSA strains ${ }^{7}$. Furthermore, CA-MRSA strains show, in average, higher in-vitro expression of PSMs, especially of the cytolytic PSMa peptides ${ }^{38}$. Together with the results of the animal models described above, these findings indicate that PSM peptides contribute to a major extent to the high virulence potential of CA-MRSA and probably also that of other virulent $S$. aureus strains. 
S. epidermidis is much less cytolytic than S. aureus ${ }^{16}$. Accordingly, the levels of cytolytic PSMs produced by $S$. epidermidis are very low. Instead, non-cytolytic PSM $\beta$ peptides are expressed at much higher levels in S. epidermidis than in S. aureus ${ }^{16}$. In that regard, it is interesting that $S$. epidermidis encodes a strongly cytolytic PSM peptide, PSM $\delta$, but PSM $\delta$ expression levels are very low ${ }^{16}$. The expression patterns of PSMs were analyzed in over 30 S. aureus strains covering many different lineages and more than 300 strains of $S$. epidermidis and always showed a similar pattern ${ }^{29}$. The PSM production pattern thus seems to reflect the "lifestyle" of a staphylococcal species, with pronounced production of the strongly cytolytic PSMs, as measured in culture filtrates, being limited to S. aureus. This also suggests that the different approaches of $S$. epidermidis and $S$. aureus to causing human disease may be, in addition to an overall paucity of toxins in S. epidermidis as compared to S. aureus $^{4}$, to a large extent a result of adaptation of biological activities within the PSM toxin family.

While the PSMa peptides of $S$. aureus have a demonstrated key role in pathogenesis 7, 8, 39, it is not yet clear which role cytolysis plays in that situation. Binding to lipoproteins in human serum was found to diminish the cytolytic capacity of PSMs ${ }^{40}$. While PSMs may still exhibit cytolytic ability in other extracellular environments, such as in a skin abscess, this finding suggests that cytolytic PSMs exert their contribution to pathogenesis to a large extent in the intracellular environment. In fact, several studies demonstrated that PSMa peptides of S. aureus facilitate neutrophil killing after phagocytosis ${ }^{34,41,42}$ (Fig. 4), and very recently, within osteoblasts ${ }^{39}$. Owing to the phenomenon of "diffusion sensing", the Agr system was predicted to be strongly expressed in the neutrophil phagosome ${ }^{43}$, leading to strong expression of the Agr-regulated PSMs. However, the stringent response appears to be the primary signal triggering up-regulation of PSMs in that context ${ }^{42}$, rather than Agr.

Traditional vaccines work by enhancing the antibody-mediated uptake of bacteria by phagocytes. However, this is not correlated with bacterial survival after phagocytosis, which is what ultimately determines the outcome of an infection. Thus, the pronounced capacity to kill phagocytes after uptake, a hallmark of virulent $S$. aureus such as CA-MRSA ${ }^{35}$ and now mechanistically linked to PSMs ${ }^{34,41,42}$, may explain at least in part why attempts to develop traditional $S$. aureus vaccines have failed ${ }^{44}$.

\section{PSMs and biofilm development}

Bacterial biofilms have a characteristic, spongy structure with channels that are important for the delivery of nutrients to deeper layers, and thus the survival of cells in biofilms. Cell density-dependent gene regulation (quorum-sensing), mediated by the Agr system in staphylococci, has long been implicated as a regulatory mechanism governing the formation of the channel-containing biofilm structure ${ }^{45-49}$. However, the underlying molecular factors have remained elusive. Recent research indicates that bacterial biofilms are structured by the activity of surfactant-like molecules and in staphylococci, the surfactants PSMs ${ }^{22,} 23$ (Fig. 4). In S. aureus, all PSMs have biofilm-structuring activities, indicating that - as expected they influence biofilm development via their shared physico-chemical properties ${ }^{22}$. PSM expression can also lead to biofilm dispersal, i.e. the detachment of cells or cellular clusters 
from biofilms, which is a key mechanism leading to the systemic dissemination of biofilm infection $^{7,22}$.

Interestingly, some $S$. aureus PSMs were reported to form fibril-like structures under specific in-vitro culture conditions, leading to enhanced biofilm accumulation ${ }^{50}$. At first glance, this observation appears to contradict the impact of PSMs on biofilm structuring and dispersal, but it is possible that the role of PSMs in biofilm development in vitro and in vivo is multi-faceted, combining several different modes of action.

\section{Antimicrobial activities}

Some PSMs such as S. epidermidis PSM $\delta$ and $\delta$-toxin may be antimicrobial, including against Streptococcus pyogenes ${ }^{51}$ (Fig. 4). However, the fact that high concentrations of PSMs are needed to detect such activity indicates that PSMs have not evolved to become antimicrobials. Proteolytic processing strongly increases the antimicrobial activities of $S$. aureus PSMa1 and PSMa2, which in their unprocessed form have little antimicrobial activity ${ }^{52}$. Whether these antimicrobially active derivatives of PSMa1 and PSMa2 occur in vivo is not known. In general, S. pyogenes appears to be most sensitive to the antimicrobial activity of PSMs, the molecular reasons of which are not understood. Potentially, PSMs may thus contribute to bacterial interference in vivo, in particular with streptococci. Notably, staphylococci are protected from PSM antimicrobial activities by the Pmt secretion system, which confers immunity to the PSM-producing organism in addition to PSMs produced by potential co-colonizers ${ }^{34}$.

\section{PSMs and immunomodulation}

PSMs are cytolytic for neutrophils in the micromolar range. At nanomolar concentrations, they stimulate leukocytes and initiate pro-inflammatory responses including neutrophil chemoattraction, activation, and the release of IL- $8^{7,11}$. Therefore, PSMs can be regarded as "pathogen-associated molecular patterns" (PAMPs). It is likely that the neutrophil-attracting properties of PSMs are important in local S. aureus infections and contribute to inflammation (Fig. 4). Leukocytes sense PSMs via formyl-peptide receptor 2 (FPR2) ${ }^{11}$, which had not previously been implicated in antibacterial host defense ${ }^{53}$. Unlike the paralogous FPR1, which responds to all bacteria by binding bacteria-specific formylated peptides, FPR2 has very low affinity for formylated peptides and responds to certain human peptides involved in chronic inflammation ${ }^{53}$. Of note, PSMs were found to be among the most potent of all known FPR2 agonists ${ }^{11}$. Since this activity is shared by all PSMs despite very low sequence similarity, FPR2 may sense the amphiphatic, a-helical structure of PSMs rather than a specific amino acid sequence motif. Among other bacterial genera FPR2 only responded to certain enterococcal strains ${ }^{54}$ and Listeria monocytogenes ${ }^{55}$, albeit activation seems to be much lower than that exerted by staphylococci ${ }^{54}$. However, PSM-related genes and the PSM exporter gene locus pmt are only found in staphylococcal genomes, indicating that those Gram-positive pathogens secrete other types of ligand peptides.

The response of FPR2 to culture filtrates of different staphylocococcal species correlated well with the virulence potential and level of PSM release by the various strains - it was very strong toward highly pathogenic CA-MRSA, moderate toward HA-MRSA and 
opportunistic pathogens such as S. epidermidis and Staphylococcus saprophyticus, and hardly detectable toward commensals such as Staphylococcus auricularis ${ }^{17}$. Therefore, it was proposed that FPR2 not only detects PAMPs but also monitors the invader's pathogenicity to appropriately adjust the immune response. In line with this notion, inhibition of the likely mouse FPR2 ortholog, mFpr-Rs2, abrogated neutrophil influx in local infections caused by PSM-producing $S$. aureus USA300 or toward locally injected PSM peptides ${ }^{11}$.

Dendritic cells (DCs), which connect innate and adaptive immunity, also express FPR2 and are attracted by PSMs. Intriguingly, PSMs induced a tolerogenic phenotype in DCs, which was characterized by reduced antigen endocytosis and inhibition of the release of proinflammatory cytokines but increased IL-10 secretion ${ }^{56}$. As a consequence, PSM-treated DCs inhibited Th1 differentiation and induced regulatory $\mathrm{T}$ cells, thereby probably contributing to the immune evasion capacities of highly virulent S. aureus (Fig. 4). However, the latter activities appeared to be FPR2-independent, suggesting that either interactions with other receptors or PSM-dependent perturbation of host cell membranes mediate the anti-inflammatory activity of PSMs in DCs.

The various and partly contrasting ways in which PSMs affect leukocyte functions raise the question how these activities interact and if the pathogen or the host profits from a particular interaction. When only small numbers of bacteria initiate a local infection, the low amounts of secreted PSMs are likely to be in the range that is sensed by FPR2, eliciting neutrophil influx and chemokine production. This process probably contributes to the clearance of infection caused by staphylococcal strains with low virulence potential. In contrast, highvirulence strains with strong PSM expression subsequently destroy phagocytes and modulate the activity of immigrating DCs, thereby subverting innate and adaptive immune responses.

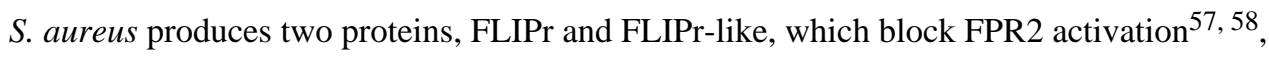
emphasizing the importance of FPR2 for the host during S. aureus infection. However, the human lipid mediator lipoxin can also block FPR $2^{59}$, suggesting that the host needs to control FPR2 activation. Furthermore, reactive oxygen species produced by activated neutrophils may abrogate the immunomodulatory effects of PSMs ${ }^{60}$. The exact biological role of host interference with PSM immunomodulatory activities remains to be elucidated.

\section{Targeting PSMs for drug development}

The strong impact that PSMs, especially PSMa peptides, have on the development of acute forms of $S$. aureus disease identifies them as promising targets for drug development. Several routes of using PSMs as drug targets can be envisaged.

First, PSMs could be used as antigens in active vaccination approaches. They offer the advantage of being secreted and crucial to virulence. Similar efforts are being undertaken using other important secreted toxins of $S$. aureus, such as a-toxin ${ }^{61}$. However, these approaches are based on the assumption that efficient protective memory against $S$. aureus can be developed, which remains a challenge for vaccine development. 
Second, PSMs could be targeted by monoclonal antibodies (mAbs). For the reasons outlined above, mAb-dependent facilitation of opsonophagocytosis may not lead to enhanced killing of $S$. aureus. However, mAbs may eliminate PSM toxicity by sequestration. For high efficacy of an anti-PSM mAb formula, the mAbs would need to work against the most cytolytic PSMs but it is unlikely that mAbs can be developed that react with all PSMa peptides, owing to the lack of epitope similarity. However, potentially it could be enough to target the main cytolysin, PSMa3. Furthermore, this method will certainly not be able to efficiently attack PSMs that are produced inside the neutrophil phagosome, because it is unlikely that sufficient amounts of sequestering anti-PSM mAbs would be ingested together with the bacteria to counteract PSM toxicity after phagocytosis. Moreover, at least in the blood, mAbs may not add much to the effect lipoproteins have in sequestering PSMs. Thus, the success of a mAb approach is dependent on a strong contribution of PSMs to disease in the extracellular environment. Because the relative contribution of different mechanism of PSM action to disease development is yet poorly understood, these considerations indicate the necessity of further research in that area.

Third, the discovery of the Pmt secretion system could facilitate the development of strategies to interfere more efficiently and broadly with PSM production. Targeting Pmt would have the benefits of abolishing production of all PSMs simultaneously, in addition to directly causing cell death. Drugs blocking the Pmt transport function might even work on all PSM-producing species, as the Pmt system is well conserved. Both small molecules and mAbs should be evaluated as Pmt blockers. As there are much fewer copies of Pmt than PSMs, it is possible that Pmt may be sufficiently blocked inside the neutrophil phagosome by mAbs that are co-ingested. Additionally, Pmt could be a target for active vaccination, given its surface location and essential role in growth and pathogenesis. Other S. aureus transport systems with or without a demonstrated function in pathogenesis are being, or have been evaluated as candidate vaccines ${ }^{62,63}$. Further development by Novartis of the transporter-targeted "Aurograb" vaccine was only stopped in clinical trials, as all other $S$. aureus vaccine candidates so far ${ }^{64}$.

Fourth, the recognition of PSMs by FPR2 might be a target for therapeutic intervention, as FPR2 blockers could reduce inflammation. However, assessing the value of such intervention requires further research delineating whether the PSM-FPR2 interaction is part of the staphylococcal pathogenesis program or whether it benefits the host.

\section{Concluding remarks}

PSMs have been recognized as key players in staphylococcal pathogenesis, influencing a series of virulence mechanisms (Fig. 4) involved in a variety of disease manifestations. There are several questions about the role of PSMs in pathogenesis and during noninfectious colonization that are still unanswered and which future research on PSMs should address. First, we do not yet understand exactly the nature of the putative "original" role of PSMs in the commensal lifestyle of staphylococci. Research in that direction will have to use better animal models of colonization that are more representative of the situation in vivo. However, such models are difficult and barely available. Second, a more detailed analysis of the mechanisms by which PSMs contribute to specific diseases will be required, including 
addressing whether and when they work in the extra- versus intracellular environment and which additional cell types are affected by PSM activities. Third, it remains enigmatic why staphylococci always produce arrays of $\alpha$ and $\beta$-type PSM peptides with largely identical activities. Moreover, the seemingly contrasting roles of PSMs during interaction with innate host defenses need specific attention. Finally, PSMs should be developed as targets for antistaphylococcal drug development, which will benefit from a deeper understanding of the functions of PSMs in pathogenesis.

\section{Acknowledgments}

This work was supported by the Intramural Research Program of the National Institute of Allergy and Infectious Diseases, U.S. National Institutes of Health (to M.O.) and by the German Research Council (SFB685, TRR34, to A.P).

\section{References}

1. Wertheim HF, et al. The role of nasal carriage in Staphylococcus aureus infections. Lancet Infect Dis. 2005; 5:751-62. [PubMed: 16310147]

2. Lowy FD. Staphylococcus aureus infections. N Engl J Med. 1998; 339:520-32. [PubMed: 9709046]

3. Klevens RM, et al. Invasive methicillin-resistant Staphylococcus aureus infections in the United States. Jama. 2007; 298:1763-71. [PubMed: 17940231]

4. Otto M. Staphylococcus epidermidis - the 'accidental' pathogen. Nat Rev Microbiol. 2009; 7:55567. [PubMed: 19609257]

5. Costerton JW, Stewart PS, Greenberg EP. Bacterial biofilms: a common cause of persistent infections. Science. 1999; 284:1318-22. [PubMed: 10334980]

6. Foster TJ. Immune evasion by staphylococci. Nat Rev Microbiol. 2005; 3:948-58. [PubMed: 16322743]

7. Wang R, et al. Identification of novel cytolytic peptides as key virulence determinants for community-associated MRSA. Nat Med. 2007; 13:1510-4. [PubMed: 17994102]

8. Kobayashi SD, et al. Comparative analysis of USA300 virulence determinants in a rabbit model of skin and soft tissue infection. J Infect Dis. 2011; 204:937-41. [PubMed: 21849291]

9. Mehlin C, Headley CM, Klebanoff SJ. An inflammatory polypeptide complex from Staphylococcus epidermidis: isolation and characterization. J Exp Med. 1999; 189:907-18. [PubMed: 10075974]

10. McKevitt AI, Bjornson GL, Mauracher CA, Scheifele DW. Amino acid sequence of a deltalike toxin from Staphylococcus epidermidis. Infect Immun. 1990; 58:1473-5. [PubMed: 2323825]

11. Kretschmer D, et al. Human formyl peptide receptor 2 senses highly pathogenic Staphylococcus aureus. Cell Host Microbe. 2010; 7:463-73. [PubMed: 20542250]

12. Hajjar AM, et al. Cutting edge: functional interactions between toll-like receptor (TLR) 2 and TLR1 or TLR6 in response to phenol-soluble modulin. J Immunol. 2001; 166:15-9. [PubMed: 11123271]

13. Liles WC, Thomsen AR, O'Mahony DS, Klebanoff SJ. Stimulation of human neutrophils and monocytes by staphylococcal phenol-soluble modulin. J Leukoc Biol. 2001; 70:96-102. [PubMed: $11435491]$

14. Otto M, O'Mahoney DS, Guina T, Klebanoff SJ. Activity of Staphylococcus epidermidis phenolsoluble modulin peptides expressed in Staphylococcus carnosus. J Infect Dis. 2004; 190:748-55. [PubMed: 15272403]

15. Periasamy S, Chatterjee SS, Cheung GY, Otto M. Phenol-soluble modulins in staphylococci: What are they originally for? Commun Integr Biol. 2012; 5:275-7. [PubMed: 22896791]

16. Cheung GY, et al. Staphylococcus epidermidis strategies to avoid killing by human neutrophils. PLoS Pathog. 2010; 6:e1001133. [PubMed: 20949069] 
17. Rautenberg M, Joo HS, Otto M, Peschel A. Neutrophil responses to staphylococcal pathogens and commensals via the formyl peptide receptor 2 relates to phenol-soluble modulin release and virulence. Faseb J. 2011; 25:1254-63. [PubMed: 21183593]

18. Kornblum, J.; Kreiswirth, B.; Projan, SJ.; Ross, H.; Novick, RP. Molecular biology of the staphylococci. Novick, RP., editor. VCH Publishers; New York, N.Y: 1990. p. 373-402.

19. Vuong C, et al. Regulated expression of pathogen-associated molecular pattern molecules in Staphylococcus epidermidis: quorum-sensing determines pro-inflammatory capacity and production of phenol-soluble modulins. Cell Microbiol. 2004; 6:753-9. [PubMed: 15236642]

20. Yao Y, Sturdevant DE, Otto M. Genomewide analysis of gene expression in Staphylococcus epidermidis biofilms: insights into the pathophysiology of $S$. epidermidis biofilms and the role of phenol-soluble modulins in formation of biofilms. J Infect Dis. 2005; 191:289-98. [PubMed: 15609240]

21. Tsompanidou E, et al. Distinct roles of phenol-soluble modulins in spreading of Staphylococcus aureus on wet surfaces. Appl Environ Microbiol. 2013; 79:886-95. [PubMed: 23183971]

22. Periasamy S, et al. How Staphylococcus aureus biofilms develop their characteristic structure. Proc Natl Acad Sci U S A. 2012; 109:1281-6. [PubMed: 22232686]

23. Wang R, et al. Staphylococcus epidermidis surfactant peptides promote biofilm maturation and dissemination of biofilm-associated infection in mice. J Clin Invest. 2011; 121:238-48. [PubMed: 21135501]

24. Donvito B, et al. Synergistic hemolytic activity of Staphylococcus lugdunensis is mediated by three peptides encoded by a non-agr genetic locus. Infect Immun. 1997; 65:95-100. [PubMed: 8975897]

25. Watson DC, Yaguchi M, Bisaillon JG, Beaudet R, Morosoli R. The amino acid sequence of a gonococcal growth inhibitor from Staphylococcus haemolyticus. Biochem J. 1988; 252:87-93. [PubMed: 3138972]

26. Cheung GY, Wang R, Khan BA, Sturdevant DE, Otto M. Role of the accessory gene regulator agr in community-associated methicillin-resistant Staphylococcus aureus pathogenesis. Infect Immun. 2011; 79:1927-35. [PubMed: 21402769]

27. Kretschmer D, Nikola N, Durr M, Otto M, Peschel A. The virulence regulator Agr controls the staphylococcal capacity to activate human neutrophils via the formyl peptide receptor 2 . J Innate Immun. 2012; 4:201-12. [PubMed: 22067547]

28. Queck SY, et al. RNAIII-independent target gene control by the agr quorum-sensing system: insight into the evolution of virulence regulation in Staphylococcus aureus. Mol Cell. 2008; 32:150-8. [PubMed: 18851841]

29. Queck SY, et al. Mobile genetic element-encoded cytolysin connects virulence to methicillin resistance in MRSA. PLoS Pathog. 2009; 5:e1000533. [PubMed: 19649313]

30. Chatterjee SS, et al. Distribution and regulation of the mobile genetic element-encoded phenolsoluble modulin PSM-mec in methicillin-resistant Staphylococcus aureus. PLoS ONE. 2011; 6:e28781. [PubMed: 22174895]

31. Kaito C, et al. Transcription and translation products of the cytolysin gene psm-mec on the mobile genetic element SCCmec regulate Staphylococcus aureus virulence. PLoS Pathog. 2011; 7:e1001267. [PubMed: 21304931]

32. Kaito C, et al. Mobile Genetic Element SCCmec-encoded psm-mec RNA suppresses translation of agrA and attenuates MRSA virulence. PLoS Pathog. 2013; 9:e1003269. [PubMed: 23592990]

33. Somerville GA, et al. Synthesis and deformylation of Staphylococcus aureus delta-toxin are linked to tricarboxylic acid cycle activity. J Bacteriol. 2003; 185:6686-94. [PubMed: 14594843]

34. Chatterjee SS, et al. Essential Staphylococcus aureus toxin export system. Nat Med. 2013; 19:3467.

35. Otto M. Basis of virulence in community-associated methicillin-resistant Staphylococcus aureus. Annu Rev Microbiol. 2010; 64:143-62. [PubMed: 20825344]

36. Loffler B, et al. Staphylococcus aureus Panton-Valentine leukocidin is a very potent cytotoxic factor for human neutrophils. PLoS Pathog. 2010; 6:e1000715. [PubMed: 20072612] 
37. Cheung GY, Duong AC, Otto M. Direct and synergistic hemolysis caused by Staphylococcus phenol-soluble modulins: implications for diagnosis and pathogenesis. Microbes Infect. 2012; 14:380-6. [PubMed: 22178792]

38. Li M, et al. Evolution of virulence in epidemic community-associated methicillin-resistant Staphylococcus aureus. Proc Natl Acad Sci U S A. 2009; 106:5883-8. [PubMed: 19293374]

39. Rasigade JP, et al. PSMs of hypervirulent Staphylococcus aureus act as intracellular toxins that kill infected osteoblasts. PLoS ONE. 2013; 8:e63176. [PubMed: 23690994]

40. Surewaard BG, et al. Inactivation of staphylococcal phenol soluble modulins by serum lipoprotein particles. PLoS Pathog. 2012; 8:e1002606. [PubMed: 22457627]

41. Surewaard B, et al. Staphylococcal alpha-Phenol Soluble Modulins contribute to neutrophil lysis after phagocytosis. Cell Microbiol. 2013; 15:1427-37. [PubMed: 23470014]

42. Geiger T, et al. The stringent response of Staphylococcus aureus and its impact on survival after phagocytosis through the induction of intracellular PSMs expression. PLoS Pathog. 2012; 8:e1003016. [PubMed: 23209405]

43. Carnes EC, et al. Confinement-induced quorum sensing of individual Staphylococcus aureus bacteria. Nat Chem Biol. 2010; 6:41-5. [PubMed: 19935660]

44. DeLeo FR, Otto M. An antidote for Staphylococcus aureus pneumonia? J Exp Med. 2008; 205:271-4. [PubMed: 18268043]

45. O'Toole GA. To build a biofilm. J Bacteriol. 2003; 185:2687-9. [PubMed: 12700246]

46. Vuong C, Gerke C, Somerville GA, Fischer ER, Otto M. Quorum-sensing control of biofilm factors in Staphylococcus epidermidis. J Infect Dis. 2003; 188:706-18. [PubMed: 12934187]

47. Vuong C, Kocianova S, Yao Y, Carmody AB, Otto M. Increased colonization of indwelling medical devices by quorum-sensing mutants of Staphylococcus epidermidis in vivo. J Infect Dis. 2004; 190:1498-505. [PubMed: 15378444]

48. Vuong C, Saenz HL, Gotz F, Otto M. Impact of the agr quorum-sensing system on adherence to polystyrene in Staphylococcus aureus. J Infect Dis. 2000; 182:1688-93. [PubMed: 11069241]

49. Yarwood JM, Bartels DJ, Volper EM, Greenberg EP. Quorum sensing in Staphylococcus aureus biofilms. J Bacteriol. 2004; 186:1838-50. [PubMed: 14996815]

50. Schwartz K, Syed AK, Stephenson RE, Rickard AH, Boles BR. Functional amyloids composed of phenol soluble modulins stabilize Staphylococcus aureus biofilms. PLoS Pathog. 2012; 8:e1002744. [PubMed: 22685403]

51. Cogen AL, et al. Selective antimicrobial action is provided by phenol-soluble modulins derived from Staphylococcus epidermidis, a normal resident of the skin. J Invest Dermatol. 2010; 130:192-200. [PubMed: 19710683]

52. Joo HS, Cheung GY, Otto M. Antimicrobial Activity of community-associated methicillinresistant Staphylococcus aureus is caused by phenol-soluble modulin derivatives. J Biol Chem. 2011; 286:8933-40. [PubMed: 21278255]

53. Ye RD, et al. International Union of Basic and Clinical Pharmacology. LXXIII. Nomenclature for the formyl peptide receptor (FPR) family. Pharmacol Rev. 2009; 61:119-61. [PubMed: 19498085]

54. Bloes DA, Otto M, Peschel A, Kretschmer D. Enterococcus faecium stimulates human neutrophils via the formyl-peptide receptor 2. PLoS ONE. 2012; 7:e39910. [PubMed: 22768166]

55. Liu M, et al. Formylpeptide receptors are critical for rapid neutrophil mobilization in host defense against Listeria monocytogenes. Sci Rep. 2012; 2:786. [PubMed: 23139859]

56. Schreiner J, et al. Staphylococcus aureus Phenol-soluble modulin peptides modulate dendritic cell functions and increase in vitro priming of regulatory T cells. J Immunol. 2013; 190:3417-26. [PubMed: 23460735]

57. Prat C, Bestebroer J, de Haas CJ, van Strijp JA, van Kessel KP. A new staphylococcal antiinflammatory protein that antagonizes the formyl peptide receptor-like 1. J Immunol. 2006; 177:8017-26. [PubMed: 17114475]

58. Prat C, et al. A homolog of formyl peptide receptor-like 1 (FPRL1) inhibitor from Staphylococcus aureus (FPRL1 inhibitory protein) that inhibits FPRL1 and FPR. J Immunol. 2009; 183:6569-78. [PubMed: 19846866] 
59. Romano M. Lipoxin and aspirin-triggered lipoxins. ScientificWorldJournal. 2010; 10:1048-64. [PubMed: 20526535]

60. Forsman H, Christenson K, Bylund J, Dahlgren C. Receptor-dependent and -independent immunomodulatory effects of phenol-soluble modulin peptides from Staphylococcus aureus on human neutrophils are abrogated through peptide inactivation by reactive oxygen species. Infect Immun. 2012; 80:1987-95. [PubMed: 22431645]

61. Kennedy AD, et al. Targeting of alpha-hemolysin by active or passive immunization decreases severity of USA300 skin infection in a mouse model. J Infect Dis. 2010; 202:1050-8. [PubMed: 20726702]

62. Anderson AS, et al. Staphylococcus aureus manganese transport protein C is a highly conserved cell surface protein that elicits protective immunity against $S$. aureus and Staphylococcus epidermidis. J Infect Dis. 2012; 205:1688-96. [PubMed: 22474033]

63. Burnie JP, et al. Identification of an immunodominant ABC transporter in methicillin-resistant Staphylococcus aureus infections. Infect Immun. 2000; 68:3200-9. [PubMed: 10816464]

64. Otto M. Novel targeted immunotherapy approaches for staphylococcal infection. Expert Opin Biol Ther. 2010; 10:1049-59. [PubMed: 20528609]

65. Doshi R, Gutmann DA, Khoo YS, Fagg LA, van Veen HW. The choreography of multidrug export. Biochem Soc Trans. 2011; 39:807-11. [PubMed: 21599652] 
A
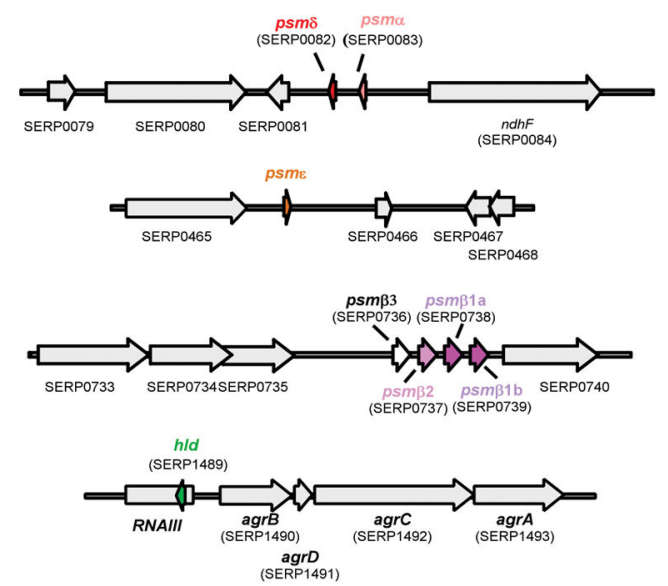

amphipathic $\alpha$-helical domain
B S. aureus
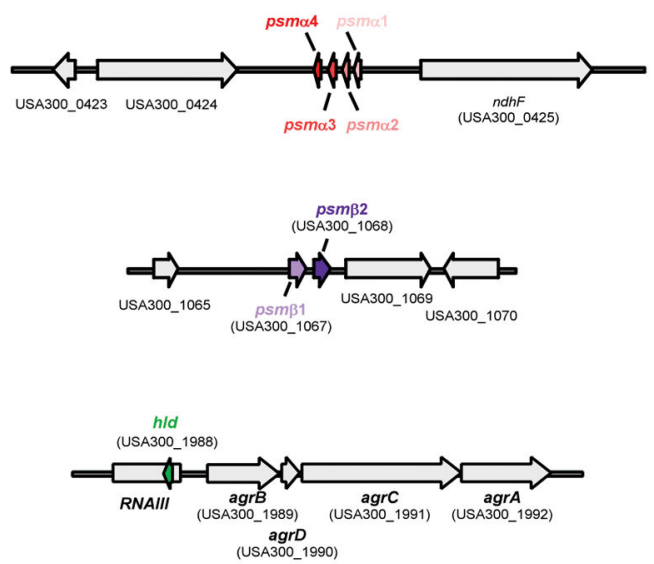

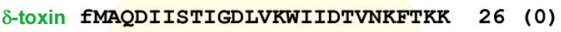

PSMl 1 fMGIIAGIIKVIKSLIEQFTGK $21(+1)$

PSM $\propto 2$ IMGIIAGIIKFIKGLIEKFTGK $21(+2)$

PSM 13 AMEFVAKLFKFFKDLLGKFLGNN $22(+1)$

$P S M O 4$ FMAIVGTIIKIIRAIIDIFAR $22(+1)$

PSM $\delta$ fMSIVSTIIEVVKTIVDIVKKFKK $23(+2)$

PSME FMFIINLVKKVISFIKGLFGNNENE 24 (0)

IMSKLAEAIANTVKAAQDQDWTKLGTSIVDIVESGVSVIGKIFGF $44 \quad(-2)$

IMEQLFDAIRSVVDAGINQDWSQLASGIAGIVENGISVISKLLGQ 44 (-4) PSM $\beta$

PSM $\beta 2$ IMTGLAEAIANTVQAAQQHDSVKLGTSIVDIVANGVGLLGKLFGF

amphipathic $\alpha$ =helical domain

D

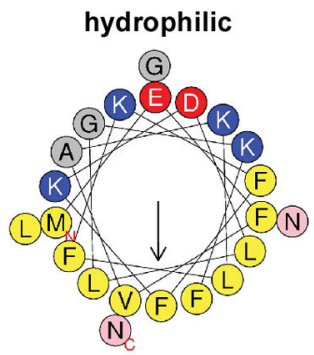

hydrophobic

Figure 1. PSM genes, amino acid sequences, and structure

$(\mathrm{A}, \mathrm{B})$ psm genes and amino acid sequences in $S$. epidermidis and $S$. aureus. Gene annotations are according to $S$. epidermidis strain RP62A and S. aureus strain USA300

FPR3757. All PSMs are secreted with an N-terminal N-formyl methionine (fM). Several atype psm genes are not annotated in staphylococcal genomes owing to their short length.

Note that the psma and $p s m \delta$ genes of $S$. epidermidis are located at a position in the genome corresponding to that of the $S$. aureus psma operon, suggesting a common ancestor of these genes. The $S$. epidermidis $p \operatorname{si} \beta$ operon contains a gene, $p s m \beta 3$, whose gene product could not be detected in culture filtrates of $S$. epidermidis strains. Some $S$. epidermidis strains, such as RP62A, may contain two identical copies of the $p s m \beta 1$ gene, resulting in higher PSM $\beta 1$ production than in strains that contain only one copy. The $\delta$-toxin (sometimes called PSM $\gamma$ ), highly similar between $S$. epidermidis and S. aureus, is encoded by the gene " $h l d$ " (for "hemolysin delta"), located within RNAIII in the Agr system. (C) Location of the psmmec gene in SCCmec elements. The psm-mec gene is found in SCCmec elements of types II, III, and VIII, in the $\mathrm{J} 2$ region next to the class A mec gene complex (with the core genes of 
the SCCmec element in the order IS431-mecA-mecR-mecI), which is characteristic for these SCCmec types. Type III is shown here as example. (D) a-helical wheel presentation of PSMa3, showing the extreme amphipathy that is characteristic of PSMs, with hydrophobic and hydrophilic amino acids found on opposite sides of the a-helix. (A-C), numbers behind amino acid sequences show peptide length and charge (in parentheses). 


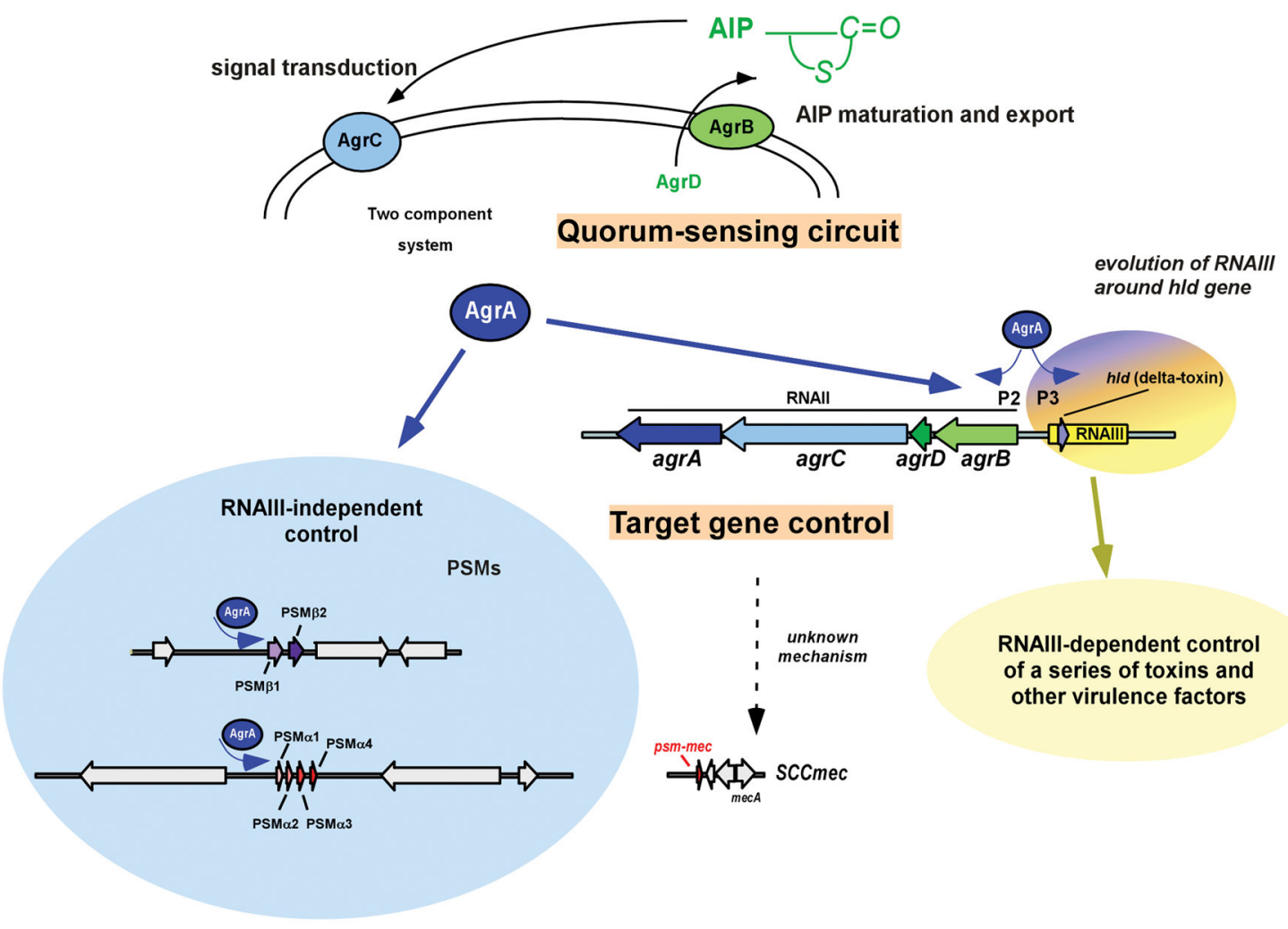

Figure 2. PSM regulation

PSMs are regulated tightly by the Agr system, a quorum-sensing (QS) system that produces and senses the presence of a post-translationally modified pheromone called AIP (autoinducing peptide). This QS circuit is shown at the top; the necessary components are encoded by the agrACDB operon. The response regulator AgrA activates transcription of the agrACDB operon in an auto-feedback loop, but also that of RNAIII, the effector molecule in charge of changing transcription levels of most genes of the Agr operon. The psma and $p \sin \beta$ genes are the only known and confirmed exceptions, as they are directly regulated by AgrA, indicating an early evolutionary link of QS and PSM production, and suggesting that RNAIII-dependent gene regulation was added later in evolution by formation of the RNAIII-encoding genetic information around the $\delta$-toxin gene, hld. The psm-mec gene is also under Agr control, but it is not known whether this occurs by direct binding of AgrA. Modified from ref. 25, with permission. 


\section{Cytolysis}

Inflammation

Biofilm structuring

\section{Immunity to PSMs of non-self}

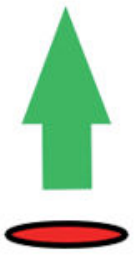

\section{Producer immunity}

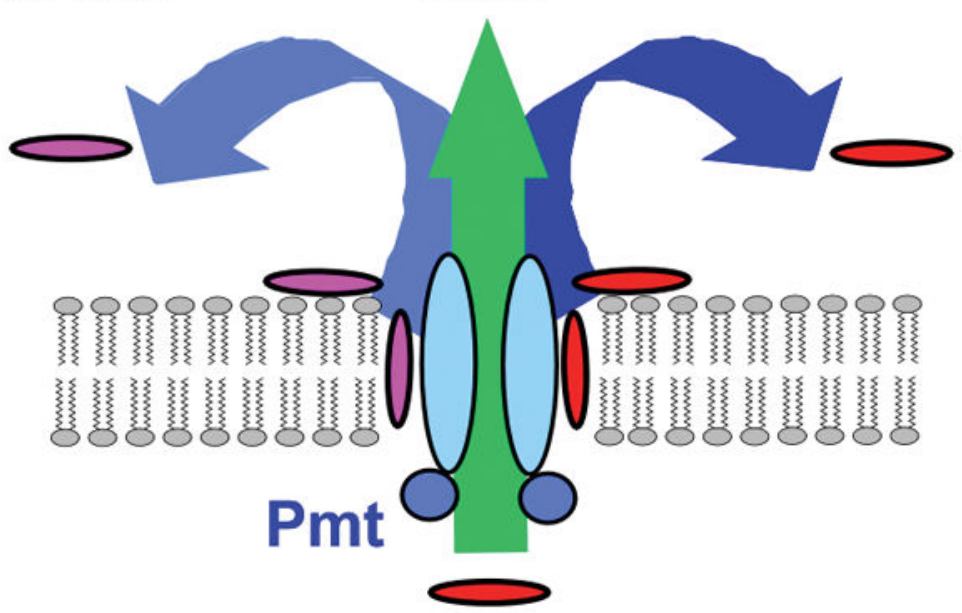

\section{Export}

Figure 3. PSM export

PSMs are secreted by the Pmt (Phenol-soluble modulin transporter) four-component ABC transporter. Presence of the Pmt transporter is crucial for PSM-mediated phenotypes, such as cytolysis, inflammation, and biofilm structuring, and immunity to PSMs of self and non-self. In analogy to other transporters that export membrane-active drugs ${ }^{65}$, the substrate is likely bound from within the membrane, using the same mechanism for PSMs originating from the cytosol or the surrounding fluid. Modified from ref. 31, with permission. 


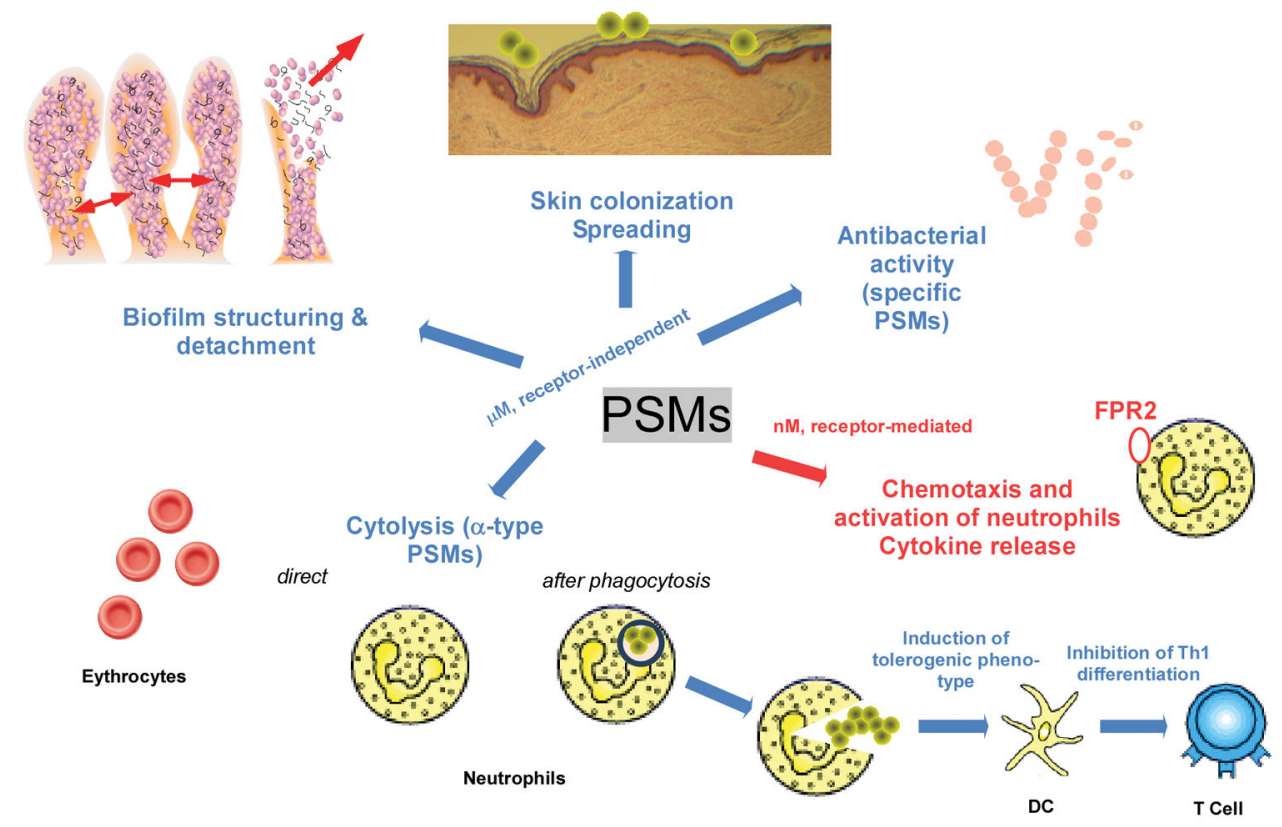

Figure 4. Overview over PSM activities

In a likely receptor-independent fashion and in the micromolar range, PSMs cause biofilm structuring and detachment, spreading on surfaces, and cytolysis. Some PSMs may also be antimicrobial, in particular towards streptococci. Cytolytic activity is found exclusively in atype PSMs. Most likely, due to the receptor-independent nature of cytolytic activity, many cell types are subject to destruction by PSMs. Erythrocytes and neutrophils are shown as examples. Lysis of neutrophils by a-type PSMs may occur after phagocytosis, making PSMs a particularly valuable weapon against elimination by innate host defense. PSMs also affect the adaptive immune system by inducing a tolerogenic phenotype in dendritic cells (DCs) and inhibiting Th1 differentiation in T cells. In the nanomolar range, all PSMs activate the FPR2 receptor, leading to neutrophil activation, chemotaxis, and cytokine release. 